\title{
Investigation of Neuroprotective Effects of Erythropoietin on Chronic Neuropathic Pain in a Chronic Constriction Injury Rat Model
}

This article was published in the following Dove Press journal: Journal of Pain Research

\author{
Kai Zhang \\ Junhao Wang \\ Haiyang $X i$ \\ Lepeng $\mathrm{Li}$ \\ Zhaohui Lou (DD
}

Department of Orthopedics, The First Affiliated Hospital of Zhengzhou University, Zhengzhou, Henan 450052, People's Republic of China
Correspondence: Zhaohui Lou Department of Orthopedics, The First Affiliated Hospital of Zhengzhou University, Zhengzhou, Henan 450052, People's Republic of China Tel/Fax +86-37I-67966I I

Email zhaohuilou123@I63.com
Introduction: Neuropathic pain is pretty common in modern society, and the treatment effect is far from satisfactory. This study aimed to find evidence of the neuroprotective effect of erythropoietin (EPO) in the treatment of neuropathic pain in a rat model of chronic constriction injury $(\mathrm{CCI})$.

Methods: A total of 30 rats were randomly divided into sham operation group, CCI group, or CCI+EPO group. The mechanical and thermal nociception thresholds are evaluated as behavioral assessments. The dorsal root ganglion cells were morphologically evaluated by hematoxylin and eosin staining, and AMPK, p-AMPK, mTOR, p70S6K, and AQP-2 proteins were compared and analyzed by Western blotting. Compared with the sham operation group, rats in the CCI group had shorter paw withdrawal threshold and paw withdrawal latency, abnormal morphology, and increased satellite glial cells.

Results: After treatment with EPO, these changes were significantly reversed. In vivo administration of erythropoietin seems to be able to regulate the expression of AQP-2 through the AMPK/mTOR/p70S6K pathway. Our study provides behavioral, morphological, and immunoblot evidence to prove the neuroprotective effect of EPO in the treatment of chronic neuropathic pain in the CCI rat model.

Conclusion: Our results indicate that EPO has the potential to treat neuropathic pain caused by peripheral nerve injury, although further verification is needed.

Keywords: erythropoietin, chronic contractile injury, dorsal root ganglia, neuropathic pain, satellite glial cells, AQP-2

\section{Introduction}

Peripheral nerve injury (PNI) is usually caused by crush injury or mechanical trauma, resulting in pain characterized by hyperalgesia and allodynia. ${ }^{1}$ It is usually spontaneous pain. Although this pain is not life-threatening, it is often unbearable, so it severely reduces the patient's quality of life. About $80 \%$ of adults have lower back or leg pain caused by neuropathic pain, ${ }^{2}$ but existing treatments cannot completely prevent pain or reduce the consequences of nerve damage. ${ }^{3,4}$ Therefore, PNI is an increasingly important public health issue.

PNI has a complicated mechanism that is still not fully understood. At present, the pathogenesis of dorsal root ganglion (DRG) injury has attracted more and more attention. A recent study reported that peripheral neuropathic pain is caused by the abnormal spontaneous activity of damaged and undamaged DRG. ${ }^{5}$ Because ganglia do not have an effective blood-nerve barrier, under certain pathological conditions, 
such as trauma or inflammation, DRG is susceptible to irritation and cause pain. ${ }^{6}$ DRG glial cells are composed of satellite glial cells. ${ }^{7}$ Neurons in sensory ganglia are surrounded by satellite ganglion cells (SGCs), and ganglion cells play a vital role in the initiation and promotion of peripheral neuropathic pain. ${ }^{8,9}$ Previous studies have shown that during the inflammatory response, SGCs can be triggered and activated by cytokines, ${ }^{10}$ which is usually closely related to neuropathic pain. Therefore, activated stem cells are considered a hallmark of PNI.

Erythropoietin (EPO) is a $30.4 \mathrm{kd}$ glycoprotein, which is the main factor regulating erythropoiesis. ${ }^{11}$ It achieves this goal by inhibiting the apoptosis of erythroid progenitor cells and supporting their clonal proliferation and differentiation into normal cells. More and more studies have shown that EPO has a protective effect on organs that are not involved in hematopoiesis. It has been confirmed that EPO has a wide range of neuroprotective effects in a variety of central and peripheral nerve injury models. ${ }^{12}$ EPO and EPO-R are widely distributed in brain neurons, Spinal cord neurons, dorsal root ganglion (DRG) neurons, Schwann cells, and axons. It can induce membrane depolarization, rapidly increase the cytoplasmic concentration of free calcium in neurons, increase dopamine synthesis and release, and increase the survival rate of central neuron cells under harmful conditions. In a purified cell culture experiment from the spinal cord of embryonic rats, Siren et $\mathrm{al},{ }^{13}$ studied the effect of recombinant human erythropoietin (rhu-EPO) on metabolically stressed motor neurons. They found that EPO increased from the density and maturity of the regenerated myelinated axons that grow at the distal end of the extrusion save the retrograde degeneration and atrophy of axons. ${ }^{14}$ They believe that EPO can protect neurons in adult spinal cord injury animal models and reduce ischemic spinal cord motor neurons of apoptosis. Buffoli et al found that the expression of AQP-2 in small-diameter DRG neurons increased after chronic constriction injury (CCI) treatment, but not in the lumbar spinal cord. These data support the hypothesis that AQP-2 expression is related to inflammatory nerve damage. ${ }^{3} \mathrm{CCI}$ is used as an animal model of chronic peripheral neuropathic pain, ${ }^{15}$ which can cause pathophysiological changes, in which ion channels and the receptor function is impaired, which causes neurochemical and ultrastructural changes.

Therefore, we want to know whether EPO has a neuroprotective effect on PNI, and whether the neuroprotective effect of EPO is achieved by regulating the expression of AQP-2. In the current study, we used rat CCI as a model of chronic neuropathic pain. ${ }^{15}$ The purpose is to find evidence of the neuroprotective effect of EPO in the CCI rat model, which may lead to a new treatment for PNI-related neuropathic pain.

\section{Materials and Methods}

\section{Animals}

A total of 30 male Sprague-Dawley rats $(240 \pm 20 \mathrm{~g}$, age 2 months) were used. All rats were housed and fed at room temperature $\left(23^{\circ} \mathrm{C}\right)$ with a humidity of $55 \% \pm 15 \%$ and a 12 $\mathrm{h}$ light/dark cycle (lights on at 7:00 a.m.). Food and water were provided adlibitum. All animals were carefully treated as per the National Institutes of Health Guidelines for the Care and Use of Laboratory Animals. All experiments were approved and supervised by the Animal Care and Use Committee of the First Affiliated Hospital of Zhengzhou University (Authorization No.: 201,822,442,015,284). The animals were randomly divided into three groups. The dose of EPO (3000 U/kg) was selected according to the previous rat studies. ${ }^{16,17}$ In the sham group $(n=10)$, the right sciatic nerve was exposed without any other treatment. The CCI group $(\mathrm{n}=10)$ underwent nerve injury as described below and had a sham injection $(0.9 \%$ saline solution, $50 \mathrm{mg} / \mathrm{kg})$ every day after surgery. The CCI + EPO group $(n=10)$ also underwent nerve injury, and the rats have then treated with EPO $(50 \mathrm{mg} / \mathrm{kg})$ injections every day after surgery.

\section{Surgical Procedures}

The CCI models were established according to the methods described in a previous study. ${ }^{15}$ Briefly, the rats were anesthetized with tiletamine/zolazepam (Zoletil WK001, Virbac, France), $50 \mathrm{mg} / \mathrm{kg}$ intraperitoneally. The right sciatic nerve was exposed at the level of the mid-thigh, and the connective tissue around the nerve was cleared. Four ligatures were then tied around the nerve at 1-2 mm intervals using $4-0$ chromic gut suture material.

\section{Behavioral Assessment}

To assess for pain, mechanical and thermal nociceptive thresholds were examined as previously described. ${ }^{18}$ To examine the mechanical nociceptive threshold, animals were placed individually into a small plastic cage with an open wire mesh bottom. Before testing, rats were left in the test cages for 30 min until their grooming and exploratory behaviors ceased and all four paws were placed on the bottom. V on Frey filaments were applied vertically to 
the plantar surface of the paw with an upward force just sufficient to bend the microfilament. We recorded the stimulus intensity at the time of foot and leg reactions, including foot reflexes, leg-stripping, and leg-turning, at intervals of 10 seconds per stimulus. The paw withdrawal threshold (PWT) index was recorded and averaged over five measurements. For thermal nociceptive thresholds, animals were placed in an acrylic box with a transparent glass plate and irradiated with radiant heat on both hind paws. The leg lift avoidance time was counted as the paw withdrawal latency (PWL). The stimulus interval was 5 min, and the average of three measurements was calculated. The behavioral assessments were performed 1 day before surgery and 3, 7, 14, and 21 days postsurgery. Animals were kept in the testing chambers for 30 minutes before each measurement to ensure they were accustomed to the test environment. After the behavioral tests, all animals were submitted to the morphological experiments.

\section{Morphology}

Hematoxylin and eosin staining was used to perform a morphological examination on the evidence of nerve damage in 5 DRG tissues. Rats in each group were anesthetized with triamine/zolapam $(50 \mathrm{mg} / \mathrm{kg})$ on day 7 $(\mathrm{n}=5)$ or day $21(\mathrm{n}=5)$ after surgery. Rats in each group were anesthetized with tiletamine/zolazepam $(50 \mathrm{mg} / \mathrm{kg})$ either on day $7(n=5)$ or day $21(n=5)$ after surgery. They were transcardially perfused with saline, followed by $250 \mathrm{~mL}$ of 4\% paraformaldehyde (PH0427, Phygene, Fujian, China) in phosphate-buffered saline (PBS, $0.1 \mathrm{M}$, $\mathrm{pH}$ 7.4). DRG tissues from L4 to L6, corresponding to sciatic afferent fibers, were removed and fixed with $4 \%$ paraformaldehyde in PBS ( $\mathrm{pH}$ 7.4) for $4 \mathrm{~h}$. After rinsing, the DRG tissues were processed and embedded in paraffin. Some tissue sections were examined after standard HE staining. All the sections were observed and quantitatively analyzed using a slice-analyzing system including an optical microscope (Nikon Ni-E, Japan) and the Image J 2.10 software package. The numbers of surrounding SGCs in each of five consecutive sections were calculated. The edematous DRG neurons, surrounding SGCs, and the abnormal cell aggregates were identified according to the cellular morphology by the same experienced researcher blinded to the treatments of groups.

\section{Immunoblotting Analysis}

21 days after the surgical procedures all the animals were killed; the spinal cord (lumbar L4-L6 segment) and dorsal root ganglia (DRG) L4-L5-L6 corresponding to the sciatic afferent fibers of each animal were removed and immediately frozen in liquid nitrogen and stored at) $80^{\circ} \mathrm{C}$ until use. The tissues were homogenized in a buffer system and centrifuged at $13,254 \mathrm{~g}$ at $4^{\circ} \mathrm{C}$ for $15 \mathrm{~min}$, and the supernatants were processed using immunoblotting analysis. Protein concentration was assessed using Albumin Standards (Pierce, Rockford, IL, USA) according to the manufacturer's instruction. Twenty micrograms of the samples were analyzed by $10 \%$ SDS-PAGE and electro-transferred to a nitrocellulose membrane (pore size $0.45 \mathrm{~lm}$; BioRad) by wet blotting ( $100 \mathrm{~V}$ for $1 \mathrm{~h}$ ). The membrane was blocked with $5 \%$ non-fat dry milk in Tris-buffered saline Tween 20 (TTBS) at $4^{\circ} \mathrm{C}$. After washing with TTBS, proteins were exposed overnight at $4^{\circ} \mathrm{C}$ to rabbit polyclonal anti-AQP-2 antibody (Bioss, bs0261R) diluted 1: 1000. These were detected using a proper biotinylated secondary antibody (Bioss, Beijing, China) and an avidin-peroxidase complex according to the manufacturer's instructions (ABC kit; Dakopatts), with a solution of $0.05 \%$ DAB (3,3-diaminobenzidine tetrahydrochloride) and $0.03 \%$ hydrogen peroxide. For the quantitative analyses, the bands were evaluated as IOD, using GEL PRO 3.1 software, and the data obtained were statistically examined.

\section{Statistic Analysis}

Data were analyzed using SPSS software (V 19.0, IBM, USA). All data are reported as mean \pm standard deviation. All statistical results were tested on both sides. The normality of distribution and homogeneity of variance tests were performed first. Analysis of variance followed by Bonferroni posthoc correction was then selected for multiple comparisons. $\mathrm{P}<0.05$ was considered to be statistically significant.

\section{Results}

CCI treatment significantly shortened the PWT (Figure 1A) and PWL (Figure 1B) as compared with the sham group. These changes were partly ameliorated by EPO treatment, although not to the normal levels of the sham group. The same differences between groups were present at postoperative days 3, 7, 14, and 21 (Figure 1).

In the sham group, DRG cells appeared morphologically normal (Figure 2A). No edema or abnormal aggregation or proliferation of cells was observed. In the CCI group, the cell bodies of DRG neurons were markedly edematous with evident vacuolar-like changes (Figure 2B). The cytoplasm was concentrated, and the cell body volume contracted and 

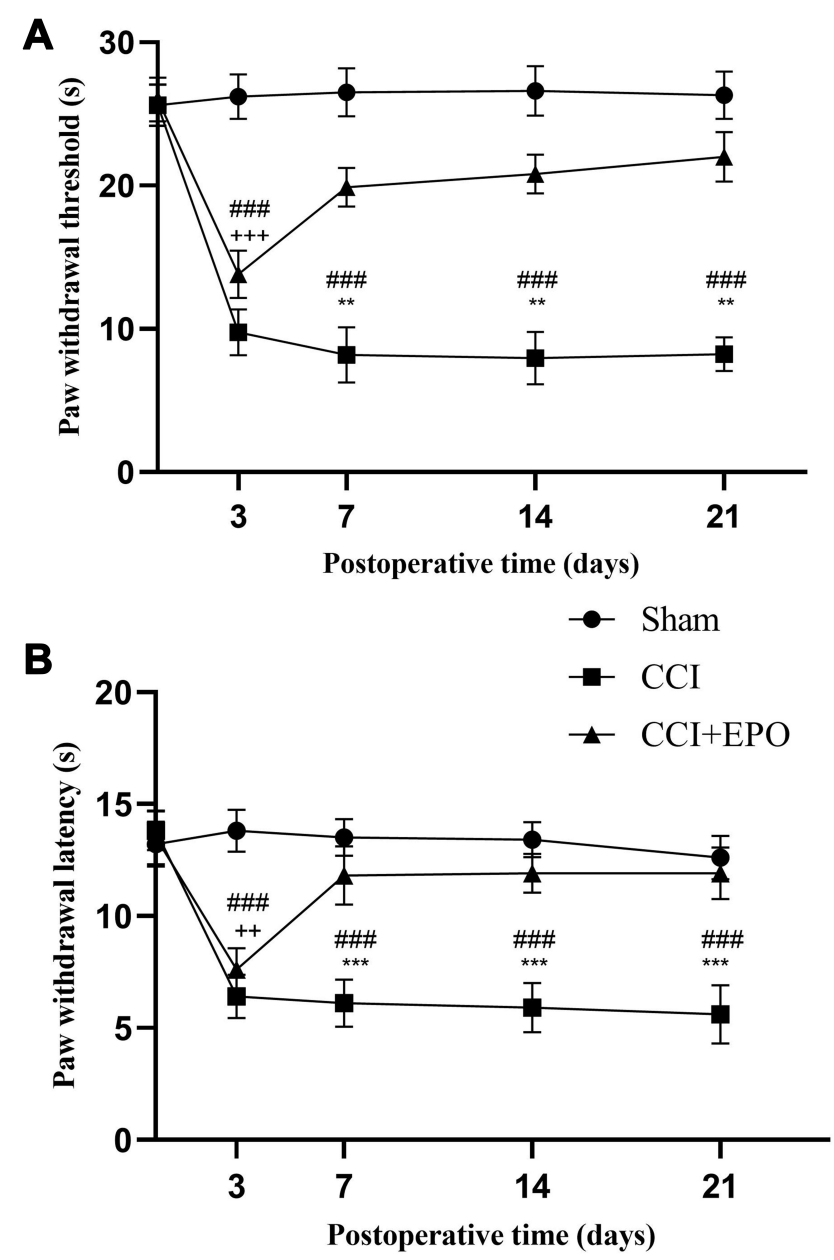

Figure I Changes in paw withdrawal thresholds (PWTs) and paw withdrawal latency $(\mathrm{PWL})$ induced by chronic constriction injury $(\mathrm{CCl})$ and its treatment with Erythropoietin (EPO).(A) The PWTs in the $\mathrm{CCl}$ group were significantly lower than those in the sham group, whereas EPO treatment significantly enhanced PWTs, although not to the level of the sham group. (B) Similar to the PWTs, the PWLs of the $\mathrm{CCl}$ group were significantly lower than those of the sham group. Treatments with EPO significantly enhanced the PWLs, although not to the level of the sham group. Sham vs $\mathrm{CCl}$, **P $<0.01$, $* * * \mathrm{P}<0.00 \mathrm{I}$. $\mathrm{CCl}$ vs $\mathrm{CCl}+\mathrm{EPO},{ }^{++} \mathrm{P}<$ $0.01,{ }^{+++} \mathrm{P}<0.001,{ }^{\# \#} \mathrm{P}<0.001$.

deformed. Occasionally, apoptotic neuronal cells were observed, and the number of SGCs was obviously enhanced. Cell aggregates were observed. Such changes indicated an enhanced inflammatory reaction associated with CCI.

In the CCI + EPO group, there was less neuronal cell body edema and fewer vacuolar changes than in the CCI group, but EPO treatment did not completely restore the tissue to a normal appearance (Figure 2C).

Counting the numbers of SGCs yielded similar results. The numbers of SGS in the CCI group were significantly higher than in the sham group, and ALA treatment partially relieved this effect, although without restoring the DRG tissues to normal. This suggests that ALA treatment partly relieves the inflammatory reaction induced by CCI (Figure 2D).
Western blot analysis showed that the expression of AQP-2 in the DRG in the sham group was close to negative staining, the AQP-2 expression in the control group was significantly increased after CCI treatment, and the expression of AQP-2 in the EPO treatment group was similar to that in the sham operation group. Calculated according to the IOD value, the staining is mainly located in the cytoplasm, while the staining of the nucleus is not obvious. This reflects some conclusions from Barbara et al (2009). That is, the expression of AQP-2 in the dorsal root ganglia of mice treated with EPO injection was lower than that of the CCI group (Figure 3A and B).

When DRG suffers from chronic constriction injury, the internal stimulation of DRG produces a large amount of AMPK and is further phosphorylated to form p-AMPK (Figure $3 \mathrm{C}$ and D). Compared with the CCI group, after EPO treatment, its AMPK phosphorylates to form p-AMPK (Figure 3E). Compared with the CCI group, the EPO treatment group had a higher ratio of AMPK phosphorylation to form p-AMPK (Figure 3E), and the expression of mTOR and its downstream p70S6K after EPO treatment was lower than that of the CCI group, but compared to the sham group, there is still a significant increase (Figure $3 \mathrm{~F}$ and $\mathrm{G}$ ).

\section{Discussion}

In this study, EPO was used to treat chronic neuropathic pain in a CCI rat model. We found that EPO treatment significantly shortened the time of PWT and PWL, improved the morphological changes of DRG neurons, reduced the aggregation and proliferation of SGCs, and reduced the expression of AQP-2. Therefore, our findings provide evidence of behavior (Figure 1), morphology (Figure 2), and immunoblotting (Figure 3), proving that we have successfully established a CCI-induced rats PNI model. We also proved that EPO can partially alleviate the pathological changes induced by CCI. Therefore, we confirmed the protective effect of EPO on chronic neuropathic pain. These findings indicate that EPO is a potential treatment for PNI-related neuropathic pain, although further verification is needed.

The CCI rats model used in this study is a classic model used to simulate chronic neuropathic pain. ${ }^{19}$ The sciatic nerve ligation can damage the peripheral nerves, so the animal may try to bite the injured leg. This abnormal behavior is considered to be a response to neuropathic pain. ${ }^{19,20}$ Because of changes in excessive pain caused by CCI, PWT and PWL may be shortened when measuring mechanical and thermal nociceptive thresholds. Our behavioral data showed 

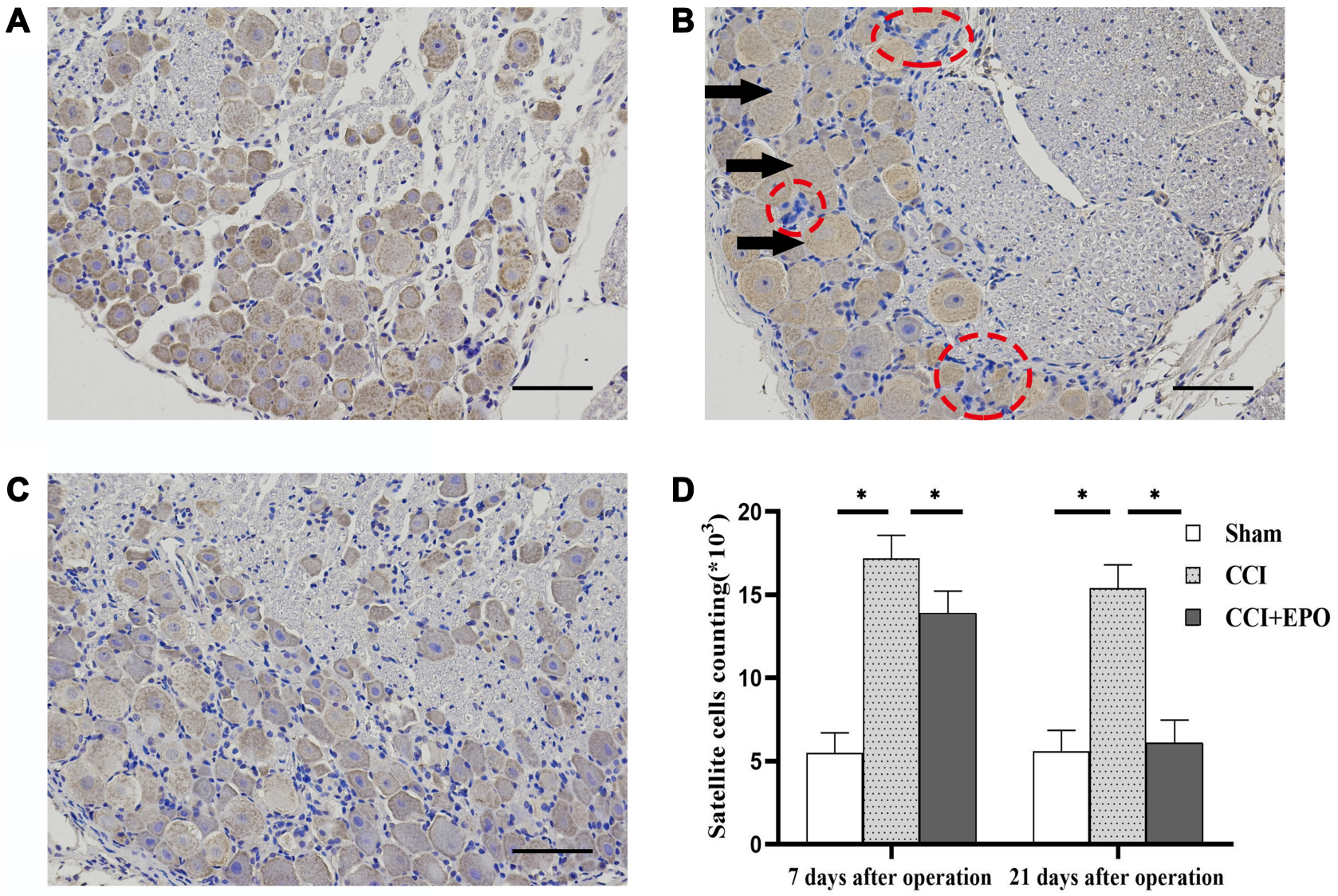

Figure 2 Morphology of dorsal root ganglia (DRG) and satellite glial cells (SGC) (hematoxylin and eosin) (A) Representative photo of DRG cells in in the sham group with normally shaped neuronal cells. (B) Representative photo of DRG cells in in the $\mathrm{CCl}$ group. The black arrows indicate edematous neurons; the red rings indicate enhanced SCGs, which form cell aggregates. (C) Representative photo of DRG cells in in the CCI + EPO. Although there are some edematous neurons and enhanced SGCs, they are fewer than in the $\mathrm{CCl}$ group. (D) SGC cells counts, significantly higher in the $\mathrm{CCl}$ group than the sham group but somewhat decreased after EPO treatment. *P < 0.05 ; scale bar $=50 \mu \mathrm{m}$.

that after CCI, the behavioral data of PWT and PWL decreased significantly. ${ }^{21}$ Together with morphological and immunoblotting evidence, our findings confirm that we have successfully established this CCI rat model. EPO treatment prolonged the PWT and PWL of the CCI+EPO group, which indicated that it reduced pain and hypersensitivity, thus confirming the efficacy of EPO treatment.

However, it is worth noting that EPO did not prevent nerve damage at the beginning of the experiment. At 3 days after the operation, the PWT and PWL of the CCI + EPO group decreased significantly and were close to the CCI group, but gradually rose back to the level close to the Sham group after 7 days. This may be due to the rapid expression of AQP $-2^{3}$ by nerve cells when the ganglion is in the acute stage of trauma, causing acute edema and inflammation, and EPO needs to gradually reduce the expression of AQP-2 through continuous administration, thereby alleviating the inflammation and inflammation of nerve cells. Edema and reduce the risk of apoptosis.
Unlike astrocytes or oligodendrocytes, SGCs are a unique type of glial cells that surround sensory neurons in the ganglia of the peripheral nervous system. Although studies have shown that SGCs play a crucial role in neuropathic pain including visceral pain $^{22}$ and peripheral neuropathic pain, ${ }^{23,24}$ the role of SGCs is not yet fully understood. Many studies have shown that various noxious stimuli may trigger the activation of stem cells. ${ }^{24,25}$ SGCs activation is considered to be a neurophysiological response to neuronal stress induced by these stimuli. ${ }^{24}$ Activated SGC usually has significant cell proliferation. ${ }^{26}$ In our CCI rat model, HE staining showed proliferation and abnormal aggregation of SGCs. Together with other morphological abnormalities of DRG neurons, we confirmed that CCI can induce nerve damage. EPO treatment reduces these physiological changes, reduces the proliferation of stem cells, reduces the edema of neurons, and makes the morphology of neurons normal. Therefore, EPO relieves the neuronal stress state, indicating that EPO has a neuroprotective effect. 
A

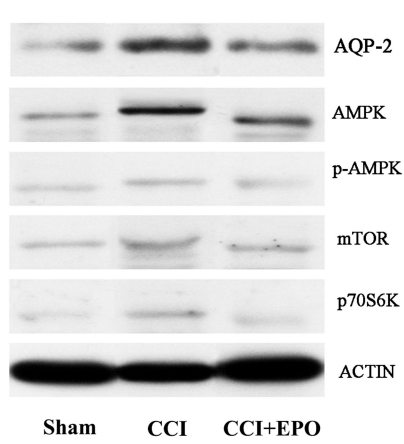

C

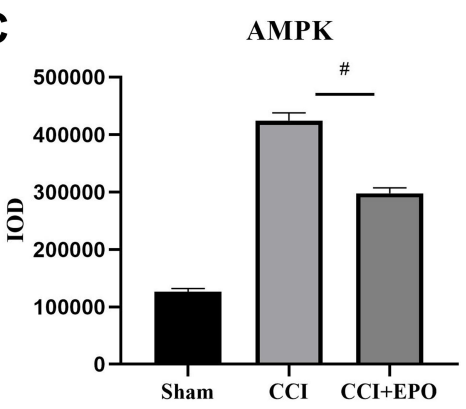

$\mathbf{E}$

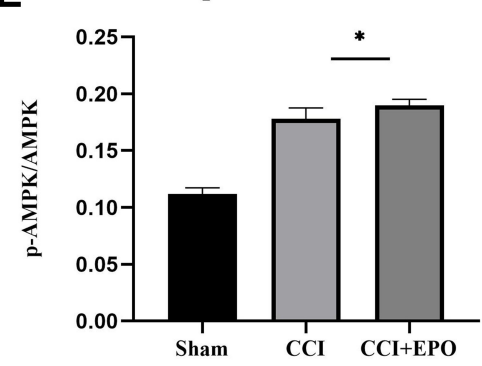

G

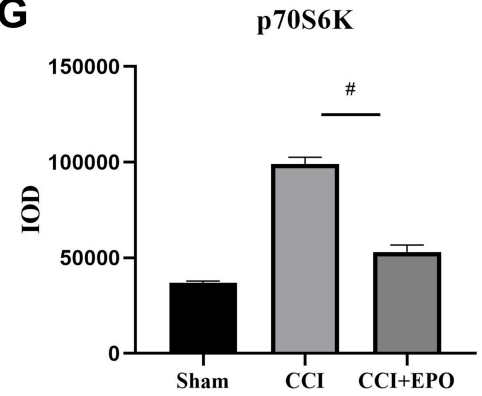

B

AQP-2

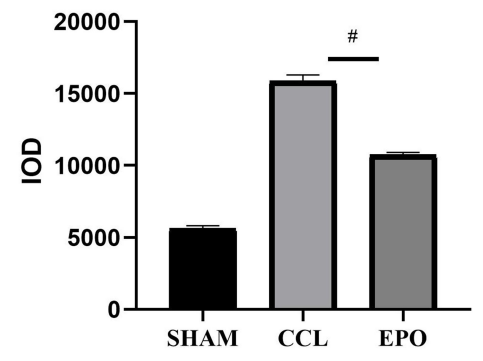

D

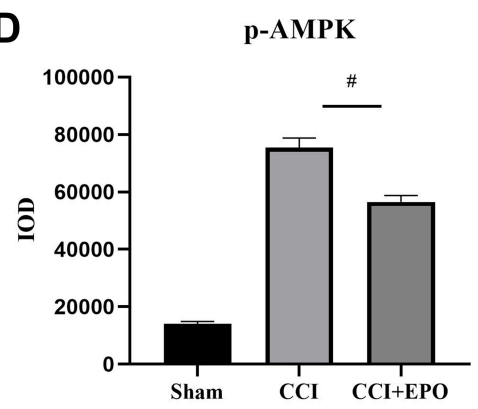

$\mathbf{F}$

mTOR

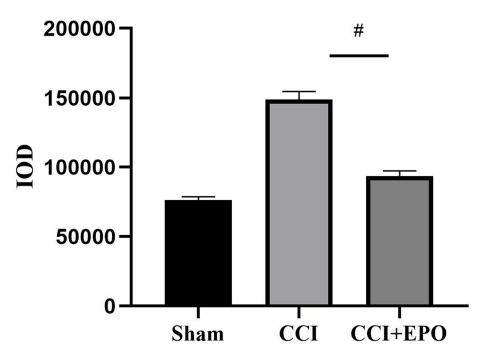

Figure 3 Western blot analysis of AQP-2, AMPK, p-AMPK, mTOR, and p70S6K expression of dorsal root ganglia (DRG). (A) Corresponding to AMPK, p-AMPK, mTOR, and $\mathrm{p} 7056 \mathrm{~K}$ bands. (B-G) are AQP-2, AMPK, p-AMPK, p-AMPK/AMPK ratio, and mTOR and p70S6K in the sham operation group, the CCl group and the CCI + EPO group were quantitative Evaluation. $\mathrm{CCl}$ vs $\mathrm{CCl}+\mathrm{EPO},{ }^{*} \mathrm{P}<0.05$, ${ }^{\#} \mathrm{P}<0.01$.

Previous studies have shown that although EPO cannot directly act on AQP-2, EPO can regulate the expression of AQP-2 through certain signaling pathways and affect cell edema and apoptosis. The findings of the AQP-2 Western blot analysis we used have also strengthened evidence. The expression of AQP-2 in the CCI group and the CCI+EPO 
group was significantly higher than that of the sham group. At the same time, it can be observed that the expression of the EPO group was significantly lower than that of the CCI group. When the CCI model was established, DRG suffered chronic constriction injury, the blood supply was lacking in its tissues, the nerve cells were in a state of ischemia and hypoxia, and the intracellular AMP/ATP ratio increased, which caused the massive production of AMPK. AMPK is an important energy sensor that can regulate cell glucose and lipid metabolism. Its expression is up-regulated under hypoxic environment, ${ }^{27}$ which makes AMPK of CCI group and EPO group significantly higher than that of the sham operation group (Figure 3C). It is worth noting that the p-AMPK/AMPK ratio of the sham group was significantly lower than that of the CCI+EPO group and the CCI group, but the CCI+EPO group had a slightly lower AMPK phosphorylation rate when AMPK and p-AMPK were lower than the CCI group. It is higher than the CCI group (Figure 3E). We speculate that the reasons may be as follows: First, systemic application of EPO can promote bone marrow hematopoietic function, promote the production of red blood cells, improve the blood's oxygen-carrying capacity, ${ }^{28}$ and relieve it to a certain extent the hypoxia of the damaged tissue reduces the AMP/ATP ratio and reduces the production of AMPK. Secondly, EPO promotes the expression of VEGE and its receptor (KDR) by activating the AMPK-KLF2 signaling pathway, ${ }^{29}$ thereby promoting vascular regeneration and development and improving the original hypoxic microenvironment reduces the production of AMPK. Thirdly, EPO may act as an activator to promote the phosphorylation of AMPK in some way and increase the ratio of p-AMPK/AMPK. A large number of literatures show that AMPK has a negative regulatory effect on its downstream mTOR/p70S6k pathway. ${ }^{29}$ When p-AMPK/AMPK increases, it will inhibit the activation of mTOR and reduce mTOR to its downstream effectors. Eukaryotic cell initiation factor $4 \mathrm{E}$ binding protein and ribosomal protein the phosphorylation of S6 kinase and 70S6k kinase inhibits protein synthesis and reduces cell energy consumption, that is, the expression of mTOR and p70S6k will be inhibited after the increase of p-AMPK (Figure 3F and G), At the same time, the synthesis process of $\mathrm{AQP}-2$ as an effector protein was also inhibited, resulting in a decrease in its expression, and to a certain extent reduced neuronal inflammatory edema and apoptosis. However, at present, how EPO promotes the intensification of AMPK into p-AMPK is still unclear. Some scholars believe that EPO can activate JAK2 and PI3K/Akt to activate or inhibit its downstream target proteins
Bad, Caspase9, NF- $\mathrm{kB}$, etc., and then regulate cell proliferation, ${ }^{30}$ differentiation, apoptosis and migration. Some studies have shown that p- AMPK is also involved in regulating voltage-gated $\mathrm{Na}+$ channels, delaying the inactivation of open channels, thereby effectively improving the electrophysiological characteristics of injured nerve cells, but the process and pathways of action need further research.

Although our study provides several different types of evidence to prove the efficacy of EPO in neuropathic pain models, its mechanism of action needs further study. Reducing the expression of AQP-2 may be a potential mechanism to inhibit neuronal apoptosis. Stevens ${ }^{31}$ found that AQP-2 is also expressed in several extrarenal sites including the peripheral nervous system. Small-diameter dorsal root ganglion neurons increase the expression of AQP-2 after chronic compression injury and strengthen the transmembrane of water molecules. After transport, it often aggravates inflammatory edema and apoptosis of nerve cells. These data support the hypothesis that AQP2 expression is related to inflammatory nerve damage. ${ }^{32} \mathrm{In}$ this study, we found that when EPO was applied systemically in CCI model rats, the expression of AQP-2 in dorsal root ganglion nerve cells decreased significantly, and cell edema and apoptosis were also reduced, indicating the important protective effect of EPO on peripheral nerves. It is related to its regulation of the expression of AQP-2. Neuronal apoptosis is a mechanism for neuropathic pain, and inhibiting cell apoptosis can improve hyperalgesia and mechanical allodynia, ${ }^{33}$ which is completely consistent with our findings in this study. All evidence strongly suggests that EPO plays a neuroprotective effect on neuropathic pain associated with PNI.

It is well known that in addition to AQP-2, EPO can also regulate many other aquaporins in the $\mathrm{AQP}$ family. In addition, many studies have shown that AQP-1 and AQP-4 are also abundantly expressed in sciatic nerve cells. ${ }^{34-37}$ The study of Hong showed that EPO treatment can prevent the down-regulation of renal AQP-4 and sodium transporter caused by ischemia. ${ }^{38}$ Annelies reported that EPO can induce the renal tubular AQP-1 and Bcl-2 in mice with acute kidney injury. ${ }^{39}$ However, the above situation is completely different to the result of the down-regulation of AQP-2 expression in DRG of CCI model rats induced by EPO in this experiment. At the same time, this experiment did not explore whether AQP-1 or AQP-4 is expressed in the DRG of CCI model rats, and whether EPO has a regulatory effect on AQP-1 or AQP-4 of CCI model rats. Therefore, future research needs further exploration on these mechanisms. 


\section{Conclusion}

In summary, our study provides evidence of behavior, morphology, and Western blot analysis of the neuroprotective effect of EPO in the treatment of chronic neuropathic pain in the CCI rat model. Our results indicate that EPO is a potentially effective treatment for neuropathic pain associated with PNI. Further clinical trial verification and exploration of treatment mechanisms will be necessary.

\section{Funding}

This research was funded by the Henan Provincial Medical Science and Technology Research Project (No. SBG J2018030).

\section{Disclosure}

The authors declare that they do not have any commercial or associative interest that represents a conflict of interest in connection with the work submitted.

\section{References}

1. Jensen TS, Finnerup NB. Allodynia and hyperalgesia in neuropathic pain: clinical manifestations and mechanisms. Lancet Neurol. 2014;13(9):924-935. doi:10.1016/s1474-4422(14)70102-4

2. van der Windt DA, Simons E, Riphagen II, et al. Physical examination for lumbar radiculopathy due to disc herniation in patients with low-back pain. Cochrane Database Syst Rev. 2010;(2):Cd007431. doi:10.1002/14651858.CD007431.pub2

3. Buffoli B, Borsani E, Rezzani R, Rodella LF. Chronic constriction injury induces aquaporin-2 expression in the dorsal root ganglia of rats. $J$ Anat. 2009;215(5):498-505. doi:10.1111/j.1469-7580.2009.01143.x

4. Schlereth T, Birklein F. The sympathetic nervous system and pain. Neuromolecular Med. 2008;10(3):141-147. doi:10.1007/s12017-007-8018-6

5. Krames ES. The dorsal root ganglion in chronic pain and as a target for neuromodulation: a review. Neuromodulation. 2015;18(1):24-32; discussion 32. doi:10.1111/ner.12247

6. Lux TJ, Hu X, Ben-Kraiem A, Blum R, Chen JT, Rittner HL. Regional differences in tight junction protein expression in the blood-drg barrier and their alterations after nerve traumatic injury in rats. Int J Mol Sci. 2019;21(1):270. doi:10.3390/ijms21010270

7. Lemes JBP, de Campos Lima T, Santos DO, et al. Participation of satellite glial cells of the dorsal root ganglia in acute nociception. Neurosci Lett. 2018;676:8-12. doi:10.1016/j.neulet.2018.04.003

8. Ohara PT, Vit JP, Bhargava A, et al. Gliopathic pain: when satellite glial cells go bad. Neuroscientist. 2009;15(5):450-463. doi:10.1177/ 1073858409336094

9. Jasmin L, Vit JP, Bhargava A, Ohara PT. Can satellite glial cells be therapeutic targets for pain control? Neuron Glia Biol. 2010;6 (1):63-71. doi:10.1017/s1740925x10000098

10. Yang F, Sun W, Yang Y, et al. SDF1-CXCR4 signaling contributes to persistent pain and hypersensitivity via regulating excitability of primary nociceptive neurons: involvement of ERK-dependent Nav1.8 up-regulation. J Neuroinflammation. 2015;12:219. doi:10.1186/s12974015-0441-2

11. Juul S. Recombinant erythropoietin as a neuroprotective treatment: in vitro and in vivo models. Clin Perinatol. 2004;31(1):129-142. doi:10.1016/j.clp.2004.03.004
12. Hernández CC, Burgos CF, Gajardo AH, et al. Neuroprotective effects of erythropoietin on neurodegenerative and ischemic brain diseases: the role of erythropoietin receptor. Neural Regeneration Res. 2017;12(9):1381-1389. doi:10.4103/1673-5374.215240

13. Sirén AL, Fratelli M, Brines M, et al. Erythropoietin prevents neuronal apoptosis after cerebral ischemia and metabolic stress. Proc Natl Acad Sci U S A. 2001;98(7):4044-4049. doi:10.1073/ pnas. 051606598

14. Toth C, Martinez JA, Liu WQ, et al. Local erythropoietin signaling enhances regeneration in peripheral axons. Neuroscience. 2008;154 (2):767-783. doi:10.1016/j.neuroscience.2008.03.052

15. Bennett GJ, Xie YK. A peripheral mononeuropathy in rat that produces disorders of pain sensation like those seen in man. Pain. 1988;33(1):87-107. doi:10.1016/0304-3959(88)90209-6

16. Campana WM, Myers RR. Exogenous erythropoietin protects against dorsal root ganglion apoptosis and pain following peripheral nerve injury. Eur J Neurosci. 2003;18(6):1497-1506. doi:10.1046/j.14609568.2003.02875.x

17. Sekiguchi Y, Kikuchi S, Myers RR, Campana WM. ISSLS prize winner: erythropoietin inhibits spinal neuronal apoptosis and pain following nerve root crush. Spine. 2003;28(23):2577-2584. doi:10.1097/01.Brs.0000096674.12519.12

18. Cobianchi S, de Cruz J, Navarro X. Assessment of sensory thresholds and nociceptive fiber growth after sciatic nerve injury reveals the differential contribution of collateral reinnervation and nerve regeneration to neuropathic pain. Exp Neurol. 2014;255:1-11. doi:10.1016/ j.expneurol.2014.02.008

19. Wall PD, Devor M, Inbal R, et al. Autotomy following peripheral nerve lesions: experimental anaesthesia dolorosa. Pain. 1979;7 (2):103-111. doi:10.1016/0304-3959(79)90002-2

20. DeLeo JA, Coombs DW, Willenbring S, et al. Characterization of a neuropathic pain model: sciatic cryoneurolysis in the rat. Pain. 1994;56(1):9-16. doi:10.1016/0304-3959(94)90145-7

21. Yin ZS, Zhang H, Bo W, Gao W. Erythropoietin promotes functional recovery and enhances nerve regeneration after peripheral nerve injury in rats. AJNR Am J Neuroradiol. 2010;31(3):509-515. doi:10.3174/ajnr. A1820

22. Lattanzi R, Maftei D, Marconi V, et al. Prokineticin 2 upregulation in the peripheral nervous system has a major role in triggering and maintaining neuropathic pain in the chronic constriction injury model. Biomed Res Int. 2015;2015:301292. doi:10.1155/2015/301292

23. Ji RR, Berta T, Nedergaard M. Glia and pain: is chronic pain a gliopathy? Pain. 2013;154(Suppl 1(0 1)):S10-28. doi:10.1016/j. pain.2013.06.022

24. Hanani M. Satellite glial cells in sensory ganglia: from form to function. Brain Res Brain Res Rev. 2005;48(3):457-476. doi:10.1016/j.brainresrev.2004.09.001

25. Lee JH, Kim W. The role of satellite glial cells, astrocytes, and microglia in oxaliplatin-induced neuropathic pain. Biomedicines. 2020;8(9):Sep. doi:10.3390/biomedicines8090324

26. Vit JP, Jasmin L, Bhargava A, Ohara PT. Satellite glial cells in the trigeminal ganglion as a determinant of orofacial neuropathic pain. Neuron Glia Biol. 2006;2(4):247-257. doi:10.1017/s1740925x07000427

27. Herzig S, Shaw RJ. AMPK: guardian of metabolism and mitochondrial homeostasis. Nat Rev Mol Cell Biol. 2018;19(2):121-135. doi:10.1038/nrm.2017.95

28. Yamanaka K, Eldeiry M, Aftab M, et al. Synergetic induction of NGF with diazoxide and erythropoietin attenuates spinal cord ischemic injury. J Surg Res. 2019;233:124-131. doi:10.1016/j.jss.2018.07.021

29. Chen GH, Li XL, Deng YQ, et al. The molecular mechanism of EPO regulates the angiogenesis after cerebral ischemia through AMPK-KLF2 signaling pathway. Crit Rev Eukaryot Gene Expr. 2019;29(2):105-112. doi:10.1615/CritRevEukaryotGeneExpr.2019029018

30. Ma C, Cheng F, Wang X, et al. Erythropoietin pathway: a potential target for the treatment of depression. Int J Mol Sci. 2016;17(5):May. doi:10.3390/ijms17050677 
31. Stevens AL, Breton S, Gustafson CE, et al. Aquaporin 2 is a vasopressin-independent, constitutive apical membrane protein in rat vas deferens. Am J Physiol Cell Physiol. 2000;278(4):C791-802. doi:10.1152/ajpcell.2000.278.4.C791

32. Kwon TH, Hager H, Nejsum LN, Andersen ML, Frøkiaer J, Nielsen S. Physiology and pathophysiology of renal aquaporins. Semin Nephrol. 2001;21(3):231-238. doi:10.1053/snep.2001.21647

33. Siniscalco D, Fuccio C, Giordano C, et al. Role of reactive oxygen species and spinal cord apoptotic genes in the development of neuropathic pain. Pharmacol Res. 2007;55(2):158-166. doi:10.1016/j. phrs.2006.11.009

34. Segura-Anaya E, Martínez-Gómez A, Dent MA. Localization of aquaporin 1 water channel in the Schmidt-Lanterman incisures and the paranodal regions of the rat sciatic nerve. Neuroscience. 2015;285:119-127. doi:10.1016/j.neuroscience.2014.10.060

35. Nesic O, Lee J, Unabia GC, et al. Aquaporin 1 - a novel player in spinal cord injury. J Neurochem. 2008;105(3):628-640. doi:10.1111/ j.1471-4159.2007.05177.x
36. Misu T, Fujihara K, Itoyama Y. [Neuromyelitis optica and antiaquaporin 4 antibody-an overview]. Brain Nerve. 2008;60 (5):527-537. Japanese.

37. Kim SM, Waters P, Vincent A, et al. Sjogren's syndrome myelopathy: spinal cord involvement in Sjogren's syndrome might be a manifestation of neuromyelitis optica. Multiple Sclerosis. 2009;15 (9):1062-1068. doi:10.1177/1352458509106636

38. Gong H, Wang W, Kwon TH, et al. EPO and alpha-MSH prevent ischemia/reperfusion-induced down-regulation of AQPs and sodium transporters in rat kidney. Kidney Int. 2004;66(2):683-695. doi:10.1111/j.1523-1755.2004.00791.x

39. De Beuf A, Hou XH, D'Haese PC, Verhulst A. Epoetin delta reduces oxidative stress in primary human renal tubular cells. J Biomed Biotechnol. 2010;2010:395785. doi:10.1155/2010/395785

\section{Publish your work in this journal}

The Journal of Pain Research is an international, peer reviewed, open access, online journal that welcomes laboratory and clinical findings in the fields of pain research and the prevention and management of pain. Original research, reviews, symposium reports, hypothesis formation and commentaries are all considered for publication. The manuscript management system is completely online and includes a very quick and fair peer-review system, which is all easy to use. Visit http:// www.dovepress.com/testimonials.php to read real quotes from published authors. 